\title{
Essential fatty acid metabolism
}

\section{By D. A. van DORP, Unilever Research, Vlaardingen, The Netherlands}

Groundnut oil, which is obtained from the fruits of Arachis hypogaea, contains a small percentage of a straight-chain saturated fatty acid (FA) with 20 carbon atoms. It was described for the first time by Gössmann (1854) and called arachidic acid. The existence of an unsaturated $\mathrm{C}_{20}$ acid with four double bonds was reported by Hartley (1909). He isolated it from liver lecithin and found that it could be converted into an octabromo-arachidic acid. Hartley was also one of the first to point out that triglycerides in depot fat contain almost entirely saturated FA, whereas in the phospholipids of the organs, polyunsaturated FA occur. He suggested that these unsaturated acids were formed from the saturated ones and that the enzymic dehydrogenations were the first steps of the energy-supplying degradation process. This concept was gradually replaced by the idea of $\beta$ oxidation, in which the degradation does not take place on the double bonds formed. Why Hartley (1909) did not give a name to the unsaturated $\mathrm{C}_{20}$ acid is not very clear, but later it was named arachidonic acid (Lewkowitsch, 1913).

Ever since $193^{\circ}$ we have known that linoleic acid is able to eliminate the deficiency symptoms which occur in rats maintained on a fat-free diet (Burr \& Burr, 1929, 1930). We also know that dietary linoleic acid is converted into arachidonic acid both in man and in other animals. Comparative tests have shown that arachidonic acid has an even stronger effect than linoleic acid. In the literature up to 1955 no reports occur of the presence of arachidonic acid in plants and vegetable oils, in contrast to reports of its presence in animal tissue. This was taken at the time as a priori proof of the conversion of linoleic acid into arachidonic acid in the animal organism. Ellis \& Isbell (1926) had already shown that in the pig, arachidonic acid is formed after feeding groundnuts, which are known to contain much linoleic acid. The finding by Thomasson (1953) that $\gamma$-linolenic acid (cis-6, cis-9, cis-12-octadecatrienoic acid) has essential FA activity, was an important step leading to a better understanding of the relationship between linoleic acid and arachidonic acid.

About 1960, it became known that $\gamma$-linolenic acid and arachidonic acid occur not only in higher animals, as was assumed up to that time, but also in the lower flora and fauna (Nichols \& Appleby, 1969). Arachidonic acid, for instance, has been demonstrated in considerable quantities in protozoa, algae, mosses and ferns, and $\gamma$-linolenic acid in fungi, flagellates, protozoa, the oil seeds of hops and hemp, Boraginaceae and Liliaceae, but we should bear in mind that the occurrence and content of FA in various organisms depend on the growing conditions, and large fluctuations within any one type may occur (Erwin \& Bloch, 1963). For unsaturated FA, such as arachidonic acid, the alga Euglena gracilis provides a 
good example. This alga is supposed to be related to the primitive Protista and may be a link between plants and animals (Korn, 1964). E. gracilis has the ability to synthesize the polyenoic acids which are characteristic of higher plants as well as those which are characteristic of higher animals. Cells of $E$. gracilis, grown while being exposed continuously to light, produce in addition to a large number of saturated FA, mainly $\alpha$-linolenic acid (cis-9, cis-12, cis-15-octadecatrienoic acid), which is not formed in cells which grow in the dark. In the absence of light, and on a poor culture medium, a large amount of arachidonic acid is formed. We know that these unsaturated FA in the cell are incorporated into the phospholipids, which in turn are used as building units of membranes. According to Rosenberg (1967), a sort of adaptation takes place under the influence of the poor growing conditions mentioned. Instead of the hydrophobic membranes which permit little contact with the environment, membranes are formed which on the basis of their structure are better able to promote the inflow of material from the aqueous environment. This enormous fluctuation in composition of the membrane needs much further study.

We know hardly anything about the exact function of these unsaturated FA in lower and higher plants. They are formed by normal FA synthesis and subsequently are dehydrogenated, as a result of which double bonds are formed, separated by methylene groups. They occur in the oils of the seeds of higher plants, and in addition to their role in membranes, they may also serve as energy sources. They are the food for the seed. In plants in which photosynthesis takes place, they are present in the cell components indispensable to that process, namely the chloroplasts.

The function of these FA is always linked with the cell membrane. It is assumed that the chain length and the extent of unsaturation of the FA in the membrane contribute to the specific physical properties of the membrane. It is not known whether unsaturated FA in plants have any other function. No specific processes have been found in which they play a part.

In the animal kingdom, the situation is different. I have already referred to the significance of linoleic acid and arachidonic acid as essential FA in mammals, but also in the lower animals there are clear indications that specific FA are essential. In the cabbage interlooper (Trichoplusia $n i$ ), for instance, $\alpha$-linolenate was shown to be an essential nutrient which could not be replaced by linoleate (Chippendale, Beck \& Strong, 1964). Might it be possible that $\alpha$-linolenate is only effective after conversion into a biologically active compound? (Christ \& van Dorp, 1972). In the animal kingdom the polyunsaturated FA have structural as well as other functions. Structurally they are important because of the physical properties which they give to membranes. They may also maintain various enzymes in these membranes in such a state that the enzymic active sites are exposed. They play a role in lipid transport and are part of certain enzymes with a lipoprotein character. This sounds very similar to what has been said about the plants. All these functions are carried out by acids with a great variation in structure. But the $n-6$ types of acids, such as linoleic acid and the dihomo- $\gamma$-linolenic acid (cis-6, cis-9, cis-1 2-eicosatrienoic acid) and arachidonic acid derived from it, take part also in a number of fascinating 
reactions leading to the formation of prostaglandins. In this paper the metabolism of these essential FA to prostaglandins will be the main area of discussion.

Prostaglandins were discovered in the I930s by von Euler. He observed, just as Kurzock and Lieb had done earlier, that human seminal plasma contains a substance which influences smooth muscles and, on injection, lowers the blood pressure in the test animal. The structures of the isolated compounds (a mixture of related compounds) were elucidated in 1962 by S. Bergstrøm and B. Samuelsson of the Karolinska Institute in Stockholm (see von Euler \& Eliasson, 1967).

In my department of the Unilever Research Laboratory in Vlaardingen we synthesized arachidonic acid radioactively labelled at specific sites and using this and other labelled compounds, we have demonstrated that dihomo- $\gamma$-linolenic acid and arachidonic acid act as precursors of prostaglandins $\mathrm{E}_{1}$ and $\mathrm{E}_{2}\left(\mathrm{PGE}_{1}, \mathrm{PGE}_{2}\right)$ respectively (Bergstrøm, Danielsson \& Samuelsson, I964; van Dorp, Beerthuis, Nugteren \& Vonkeman, 1964). In our further work we have followed the metabolic fate of the essential FA which act as precursors for prostaglandin synthesis. The enzymes which play a role in the biosynthesis of prostaglandins are bound to membranes. When trying to solubilize them from membranes, one gets the

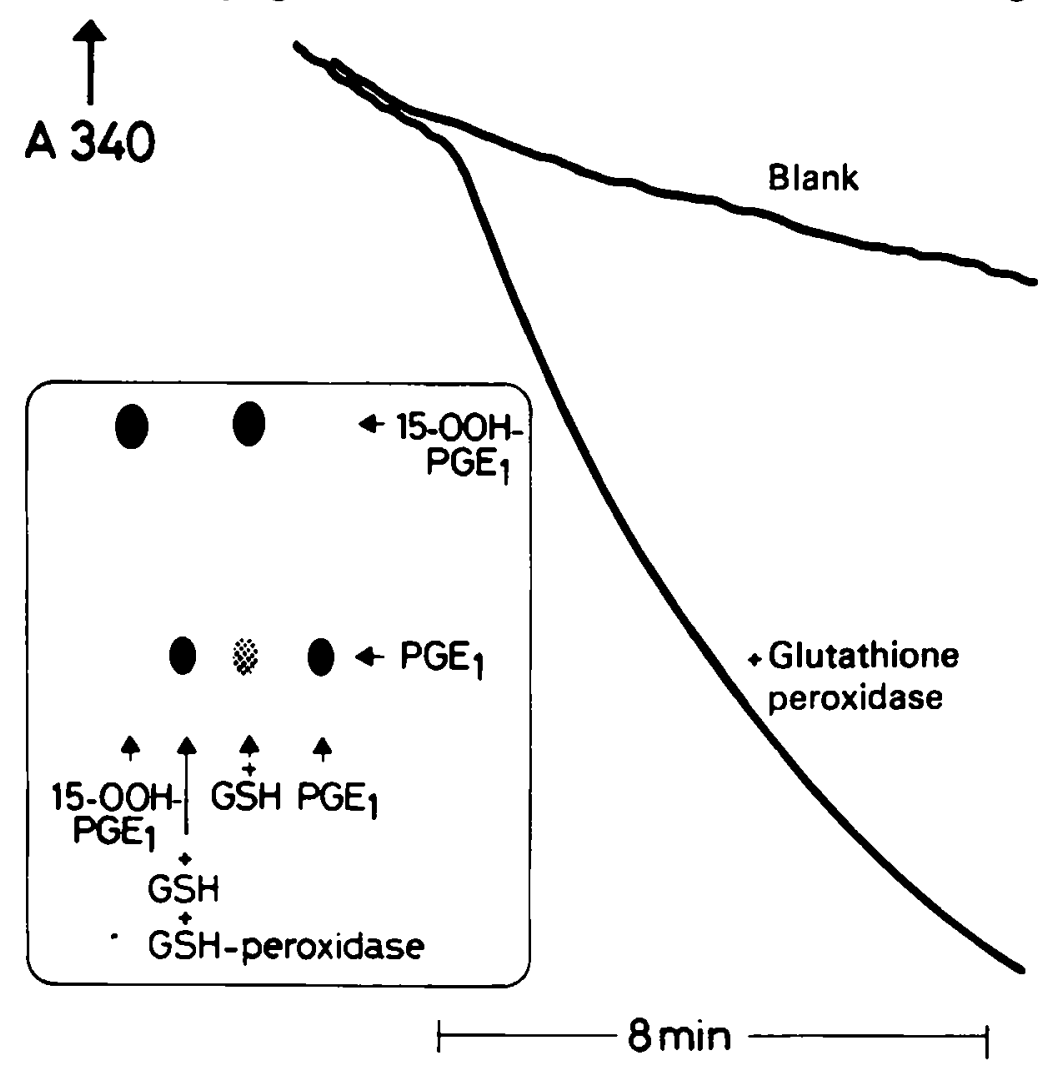

Fig. 1. The rapid enzymic reduction of 15 -hydroperoxy-prostaglandin $E_{1}$ (15-OOH-PGE I $_{1}$ by glutathione peroxidase ( $E C$ 1.11.1.9); $0.2 \mu \mathrm{mol}{ }_{15}$-OOH-PGE, $2 \mu \mathrm{mol}$ reduced glutathione (GSH), $0.6 \mu \mathrm{mol} \mathrm{NADPH}$ and $5 \mu \mathrm{mol} \mathrm{NaN}_{3}$ were incubated with glutathione reductase (EC r.6.4.2) in $2.3 \mathrm{ml} \mathrm{0.1} \mathrm{M}-\mathrm{K}_{2} \mathrm{HPO}_{4}$. $\mathrm{PGE}_{1}$, prostaglandin $\mathrm{E}_{1} ; \mathrm{A}_{340}$, extinction at $340 \mathrm{~nm}$. 
impression that it is possible to detach components from the enzyme complex which are able to catalyse certain steps of the process. As yet, however, we are still far from techniques which give pure enzymes.

Experiments with ${ }^{18} \mathrm{O}_{2}$ and mixtures of ${ }^{18} \mathrm{O}_{2}$ and ${ }^{16} \mathrm{O}_{2}$ have shown that the two oxygen atoms on the five-membered ring originate from one $\mathrm{O}_{2}$ molecule, whereas the initial reaction in the abstraction of hydrogen occurs on $\mathrm{C}-13$, as the Swedish investigators have proved so elegantly. Depending on the conditions, various products may be formed during this reaction (Nugteren, Beerthuis \& van Dorp, 1966; Hamberg \& Samuelsson, 1967).

In experiments with a homogenate from sheep vesicular glands as the enzyme source, and in the presence of glutathione, the reaction gives almost exclusively prostaglandin E (PGE). One of the enzymes involved is assumed to be glutathione peroxidase ( $E C$ I.I I.I.9). Our assumption (van Dorp, unpublished results) that this enzyme can participate is supported by the results of the experiments depicted in Fig. $r$. This figure shows, for instance, that 15 -hydroperoxy-PGE $E_{1}$ is reduced very quickly. On the basis of this experiment, an analytical method is feasible to determine labile intermediates in this system.

If glutathionine is omitted from the homogenate, a mixture of PGE and prostaglandin F (PGF) is obtained, together with by-products that point to a nonenzymic decomposition of the intermediate peroxides. More recently Hamberg, Svensson, Wakabayashi \& Samuelsson (1974) have shown that a product related to these by-products is under enzymic control. Intermediate products with an unstable endoperoxide ring have now been isolated (Hamberg \& Samuelsson, 1973; Nugteren \& Hazelhof, 1973; Hamberg et al. 1974). The reason for us to isolate them was the discovery by Vane that lung tissue of guinea-pigs produced an unstable, biologically active substance which has the ability to stimulate the contraction of rabbit aorta. Vane assumed that this substance, which he called Rabbit Aorta Contracting Substance (RCS), had something to do with the formation of prostaglandins (Piper \& Vane, 1969). Although RCS exerts a contracting effect on smooth muscular tissue, it could not be identical to any of the

Table I. Biological activities of some prostaglandin $(P G)$ derivatives (Values relative to $\mathrm{PGE}_{2}$ )

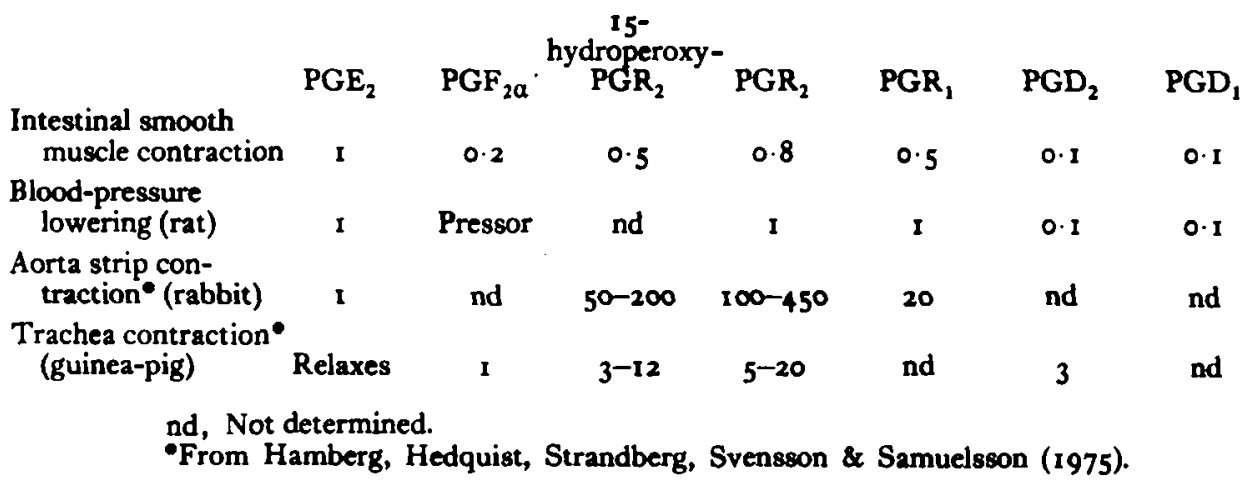


prostaglandins, because these have much greater stability. Vane therefore assumed that the endoperoxides could be identical to the RCS (Gryglewski \& Vane, 1971). After this endoperoxide became available in a pure state, it was established that its contracting effect on rabbit aortas exceeds that of the prostaglandins. Thus we have the interesting but complex situation that during this entire process the intermediate products themselves, sometimes even in nanogram amounts, possess important biological activities (Table I), although it should be noted that after certain metabolic processes inactive products are formed, which are ultimately excreted in the urine.

Kloeze (r g69), of our biological department, has established that prostaglandins, besides their effect on smooth muscle, also influence blood platelet aggregation induced by $A D P$. He found that $\mathrm{PGE}_{1}$ prevents this aggregation and that $\mathrm{PGE}_{2}$ has only a weak effect or none. There are indications that arachidonic acid itself promotes blood platelet aggregation. This was first established in human blood in vitro by Silver, Smith, Ingerman \& Kocsis (1973) and confirmed in vivo by Silver, Hoch, Kocsis, Ingerman \& Smith (1974) who injected healthy rabbits with arachidonic acid and observed that of the fifteen rabbits injected with $\mathrm{I} \cdot 4 \mathrm{mg}$ sodium arachidonate/kg body-weight, not one survived more than $3 \mathrm{~min}$. Postmortem examination showed that a considerable aggregation of blood platelets had taken place in a number of organs, particularly the lungs. One is almost forced to assume that prostaglandin synthesis in the blood platelets caused fatal thrombosis in the arteries of the lung. This was the reason for Nugteren (1975a) investigating biochemical processes of arachidonic acid in blood platelets. But, because PGE $_{2}$ did not have this effect, and neither did dihomo- $\gamma$-linoleic acid and linoleic acid

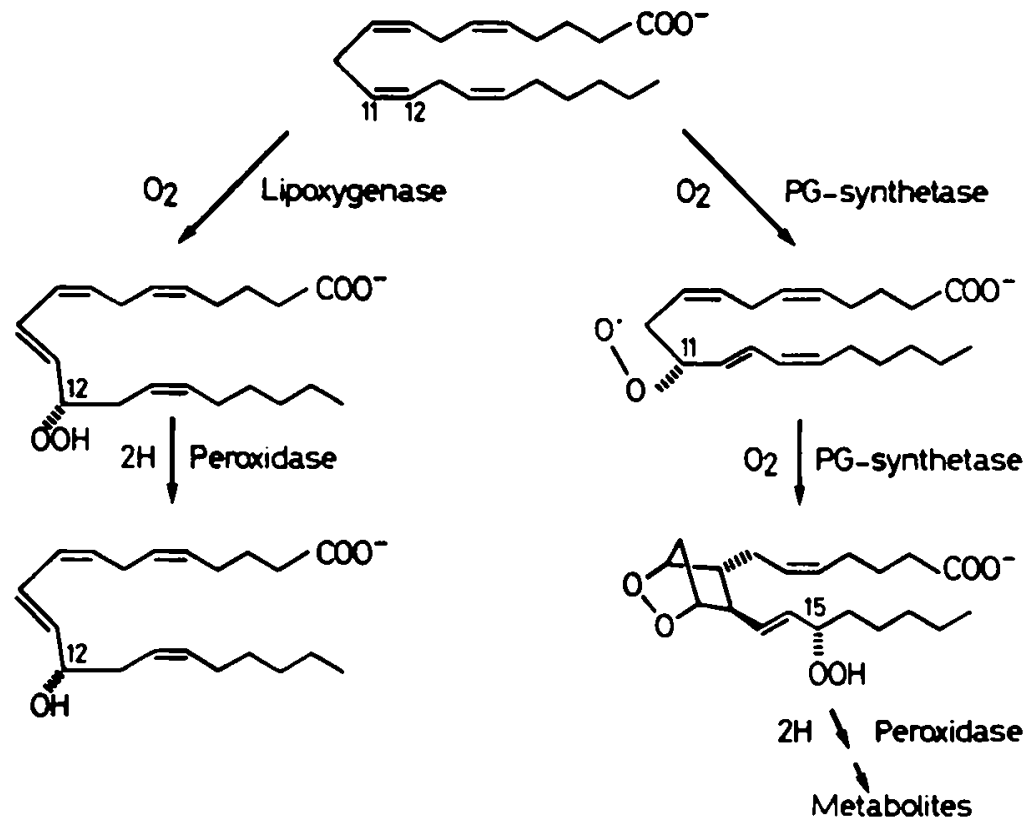

Fig. 2. The alternative pathways for enzymic conversion of arachidonate in blood platelets. PG-synthetase, prostaglandin synthetase. 
injected in an amount equal to the arachidonic acid, the investigation was continued assuming that the biologically highly active intermediate product, the endoperoxide, was responsible for the aggregation. Indeed, the observation by Willis, Comai, Kuhn \& Paulsrad (1974) that dihomo- $\gamma$-linolenic acid counteracts the thrombotic effect of arachidonic acid adds further support to the hypothesis that it is the endoperoxides $\left(\mathrm{PGR}_{2}\right.$; also called $\mathrm{PGH}_{2}$ ) which are responsible. It may be postulated that the concentrations of free arachidonic acid used were abnormally high in these experiments and it could be that high concentrations of $\mathrm{PGR}_{2}$ were formed from arachidonic acid during the fatal thrombosis. Normally, such a high concentration is apparently never achieved, but quantitative measurements of this phenomenon are still lacking and these are essential. I believe that a further study of this phenomenon can elucidate the mechanisms of such processes.

Hamberg \& Samuelsson (1974) as well as Nugteren (1975a) recently discovered in blood platelets an entirely new conversion of arachidonic acid into an oxy-acid in high yield, under the influence of a lipoxygenase (Fig. 2). This is a reaction which proceeds in the blood platelets in addition to the formation of prostaglandins; there may be up to ten times as much arachidonic acid converted to oxy-acid as to prostaglandins judging by measurements made in vitro. Mead acid (cis-5, cis-8, cis-I I-eicosatrienoic acid) has been shown to be an excellent substrate for this new enzyme (Nugteren, 1975a). Here we have another example of a large number of reactions in which the right balance is needed to ensure optimum growth and preservation of man and animals. We know only partly the danger zones and the possibilities of avoiding them. In this respect, investigations into the metabolism of essential FA and prostaglandins are important. Most important from a general dietary viewpoint are the nutrition experiments by Hornstra (1973-4), who has shown that an antithrombotic effect can be attributed to linoleic acid. We now know that prostaglandins can be converted by various reactions and that they are found eventually as inactive metabolites in the urine (Fig. 3). The total production of prostaglandin metabolites is about $1 \mathrm{mg} / 24 \mathrm{~h}$ (Nugteren, 1975b). For an adult, however, the intake of linoleic acid can be estimated at more than $10 \mathrm{~g} / \mathrm{d}$. Thus there is a factor of at least 10000 between the linoleic acid which enters the body with the food and the prostaglandin metabolites which leave the body in the urine. Hence there is an abundance of essential material. Van Dorp (1974) has speculated on the extent to which arachidonic acid and dihomo- $\gamma$-linolenic acid fulfill a multiple function: on the one hand that of precursor of $\mathrm{PGE}_{2}$ and $\mathrm{PGF}_{2 \alpha}$ or $\mathrm{PGE}_{1}$ and $\mathrm{PGF}_{1 \alpha}$ respectively and on the other hand that of essential building unit of the membranes.

The FA composition of cell membranes may change greatly, depending on the nutritional condition of the organism. This intriguing situation requires a detailed study of the physical structure of membranes. One of the requirements, then, is knowledge of the conformation of such important building-blocks as the unsaturated phospholipids. We have made a start by investigating the conformation of arachidonic acid by $\mathrm{X}$-ray analysis and nuclear magnetic resonance (NMR) spectroscopy. Extrapolation of known measurements of oleic 


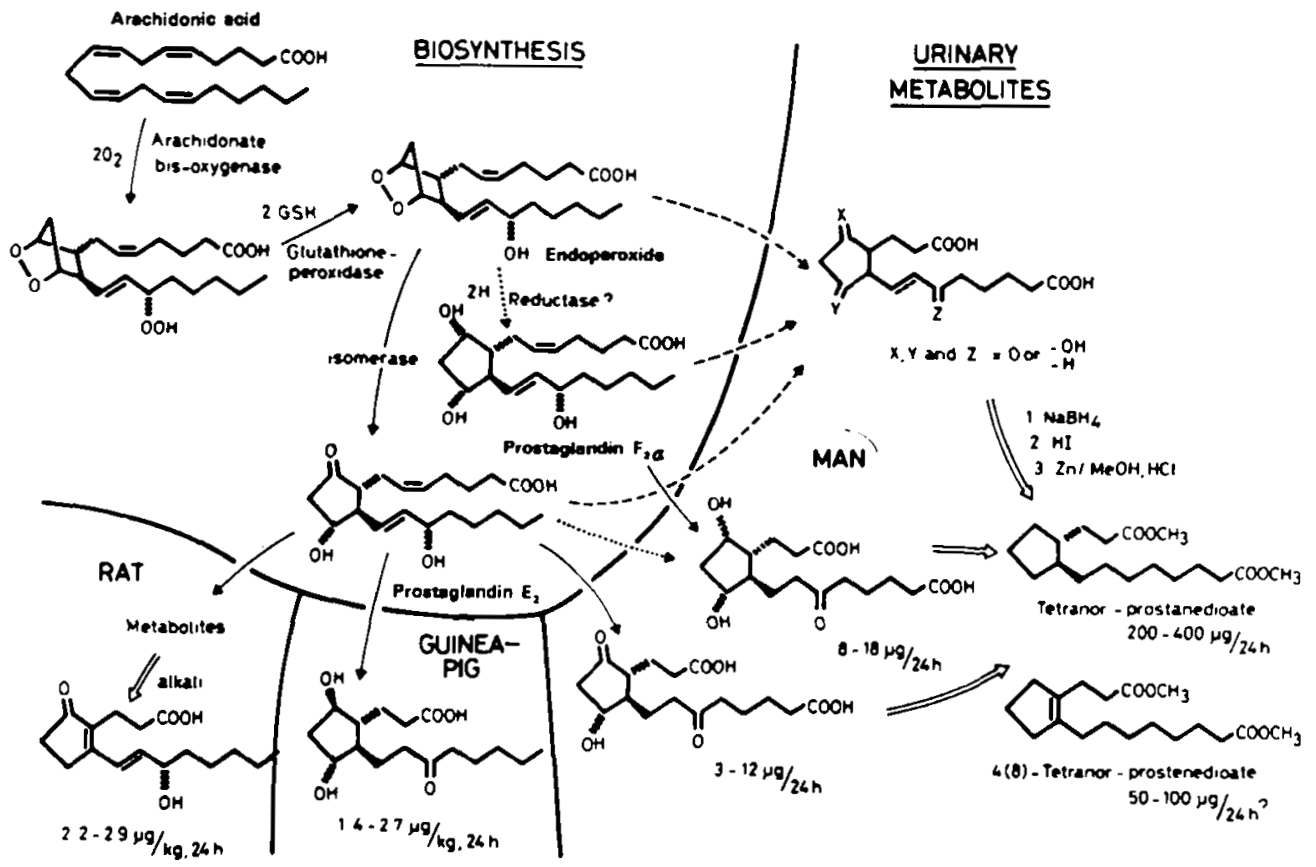

Fig. 3. Biosynthesis and metabolism of prostaglandins; $\longrightarrow$, established enzymic conversions; $\ldots . .$. , minor reaction; $\longrightarrow-\longrightarrow$, unknown pathway; $\rightleftharpoons$, chemical conversion. GSH, reduced glutathione.

acid might give a U-conformation to arachidonic acid. Powder-diffraction photographs of 1,3-dipalmitoyl-2-arachidonyl-glyceride suggest that arachidonic acid is bent and that the head-to-tail distance is about $2 \mathrm{~nm}$. A U-form would give a distance of $\mathrm{I} \cdot 2 \mathrm{~nm}$, whereas a totally stretched conformation would give $2.5 \mathrm{~nm}$.

Further information was obtained by NMR measurements from solutions in chloroform of esters of arachidonic acid and a positional isomer: trans-14arachidonic acid. Making use of europium complexes of these compounds, we found that the shifts were twice as great in the cis-14-isomer, which indicates a shorter distance between the protons of the methyl group and the Eu atom located near the ester. Here again calculations led to a distance of about $2 \mathrm{~nm}$ (van Dorp, 1974).

\section{Conclusions}

Arachidonic acid takes part in a number of complicated enzymic processes in which not only the prostaglandins but also the intermediate products of their biosynthesis have important physiological effects. The prostaglandin-synthesizing system is found both in the higher animals and in lower animals such as the corals and mussels. So far there have been no indications that this system occurs in the plant kingdom, even in those plants which are able to produce arachidonic acid. It is attractive to assume that this arachidonic acid-prostaglandin system functions as a regulator of certain membrane functions for which there is no parallel need in the plant. It is our task to study all these various reactions and their relation to 
each other. At the same time we shall try to elucidate further the conformation of arachidonic acid, which can be considered as an important building-block of the phospholipids in membranes.

\section{REFERENCES}

Bergstrøm, S., Danielsson, H. \& Samuelsson, B. (1964). Biochim. biophys. Acta 90, 207. Burr, G. O. \& Burr, M. M. (1929). F. biol. Chem. 82, 345 . Burr, G. O. \& Burr, M. M. (1930). F. biol. Chem. 86, 587.

Chippendale, G. M., Beck, S. D. \& Strong, F. M. (1964). Nature, Lond. $204,710$.

Christ, E. J. \& van Dorp, D. A. (1972). Biochim. biophys. Acta 270, 537.

Ellis, N. R. \& Isbell, H. S. (1926). F. biol. Chem. 69, 219.

Erwin, J. \& Bloch, K. (1963). Biochem. Z. 338, 436.

Gössmann, A. (1854). Anrin Phys. 89, 1 .

Gryglewski, R. \& Vane, J. R. (1971). Br. F. Pharmac. Chemother. 43, 420P.

Hamberg, M., Hedquist, P., Strandberg, K., Svensson, J. \& Samuelsson, B. (1975). Life Sci. I6, 45 I.

Hamberg, M. \& Samuelsson, B. (1967). F. biol. Chem. 242, 5336.

Hamberg, M. \& Samuelsson, B. (1973). Proc. natn. Acad. Sci. U.S.A. 70, 899.

Hamberg, M. \& Samuelsson, B. (r974). Proc. natn. Acad. Sci. U.S.A. 71, 3400.

Hamberg, M., Svensson, J., Wakabayashi, T. \& Samuelsson, B. (1974). Proc. natn. Acad. Sci. U.S.A. 71, 345 .

Hartley, P. (Igog). F. Physiol., Lond. 38, 353 .

Hornstra, G. (1973-4). Haemostasis 2, 21.

Kloeze, J. (1969). Biochim. biophys. Acta $187,285$.

Korn, E. D. (1964). Y. Lipid Res. 5, 352.

Lewkowitsch, J. I. (Igr3). Chemical Technology and Analysis of Oils, Fats and Waxes, 5 th ed., vol. I, p. 21 I. London: Macmillan \& Co.

Nichols, B. W. \& Appleby, R. S. (1969). Biochemistry, Easton 8, 1907.

Nugteren, D. H. (1975a). Biochim. biophys. Acta 380, 299.

Nugteren, D. H. (1975b). F. biol. Chem. 250, 2808.

Nugteren, D. H., Beerthuis, R. K. \& van Dorp, D. A. (1966). Recl Trav. chim. Pays-Bas $85,406$.

Nugteren, D. H. \& Hazelhof, E. (I973). Biochim. biophys. Acto 326, 448.

Piper, P. J. \& Vane, J. R. (1969). Nature, Lond. 223, 29.

Rosenberg, A. (1967). Science, N.Y. I57, I189.

Silver, M. J., Hoch, W., Kocsis, J. J., Ingerman, C. M. \& Smith, J. B. (1974). Science, N.Y. I83, 1085 .

Silver, M. J., Smith, J. B., Ingerman, C. \& Kocsis, J. J. (1973). Prostaglandins 4, 863.

Thomasson, H. J. (1953). Int. Z. VitamForsch. $25,62$.

van Dorp, D. A. (1974). Proc. Xxivth int. Congr. pure appl. Chem. 2, II7.

van Dorp, D. A., Beerthuis, R. K., Nugteren, D. H. \& Vonkeman, H. (1964). Biochim. biophys. Acta 90,204 .

von Euler, U. S. \& Eliasson, R. (1967). Prostaglandins. New York and London: Academic Press. Willis, A. L., Comai, K., Kuhn, D. C. \& Paulsrad, J. (1974). Prostaglandins 8, 509. 